\title{
Optimal Control Problems for Path Planing of AUV using Simplified Models
}

\author{
Maria do Rosário de Pinho ${ }^{1}$
}

Zahra Foroozandeh ${ }^{2}$

Aníbal Matos ${ }^{3}$

\begin{abstract}
Here we propose a simplified model for the path planning of an Autonomous Under Vehicle (AUV) in an horizontal plane when ocean currents are considered. The model includes kinetic equations and a simple dynamic equation. Our problem of interest is a minimum time problem with state constraints where the control appears linearly. This problem is solved numerically using the direct method. We extract various tests from the Maximum Principle that are then used to validate the numerical solution. In contrast to many other literature we apply the Maximum Principle as defined in [9].
\end{abstract}

\section{INTRODUCTION}

Optimal control problems for autonomous vehicles have long proved to be a useful tool for robotics and, in particular, to determine references trajectories to execute certain tasks. They have also proved to be of interest to illustrate and test many theoretical concepts of optimal control (see [1], for example). Indeed, the path planning of autonomous vehicles has received considerable attention in the last decade (see for example, [2] and reference within). In practice, computational simulations use optimal control to determine reference trajectories to be followed by the vehicle to accomplish its mission. Various models have been proposed for such simulations, with different degree of accuracy. Solving numerically optimal control problems based on accurate models for AUV's may be a hard, if not impossible, task given their complexity. Moreover, validation of the numerical solution may turn to be a enormous problem. It is however well accepted that for the task of path planning simple models capturing the main characteristics of the vehicle are good enough (see [8]).

Here we propose a simplified model to determine the path of an Autonomous Underwater Vehicle (AUV) on a horizontal plane to go from one point to a target set $T$ in the minimum time when currents are taken into account. We consider the motion using a simplified point mass model and we couple the kinetic equations of the motion with another differential equation depicting the dynamic of the vehicle. This equation involves the velocity and the thruster's force. Noteworthy, we also impose constraints on the velocity which, in control terms, reduces to the inclusion of state constraints. We solve the problem numerically using a direct approach: we first discretize the problem and then, using A

\footnotetext{
${ }^{1}$ MdR de Pinho is with Faculdade de Engenharia Universidade do Porto, SYSTEC, DEEC, Porto, Portugal, mrpinho@fe.up.pt

2 Z. Foroozandeh is with Faculty of Mathematics and Computer Science, Amirkabir University of Technology, Tehran, Iran, zahra.foroozandedaut.ac.ir

${ }^{3} \mathrm{~A}$. Matos is with INESC-TEC and Faculdade de Engenharia Universidade do Porto, DEEC, Porto, Portugal anibal@fe.up.pt
}

Mathematical Programming Language (AMPL) as the interface to the optimization solver Interior- Point optimization solver (IPOPT). Our numerical solution is validated using various tests provided by the maximum principle. Differing from other literature we use the Maximum Principle as stated in [9] where measures are associated with the multipliers of the state state constraints. In this respect, we also bring to our discussion the concept of degeneracy of the Maximum Principle, a crucial aspect in Optimal Control.

Notation: If $g \in \mathbb{R}^{m}$, the inequality $g \leq 0$ is interpreted component-wise. Also, $|\cdot|$ is the Euclidean norm or the induced matrix norm on $\mathbb{R}^{p \times q}$. The closed unit ball centred at the origin is denoted by $\bar{B}$ whereas $B$ denotes the open unit ball, regardless of the dimension of the underlying space.

For a function $h:[a, b] \rightarrow \mathbb{R}^{p}$, we say that $h \in$ $W^{1,1}\left([a, b] ; \mathbb{R}^{p}\right)$ if and only if $h$ is absolutely continuous and that $h \in L^{1}\left([a, b] ; \mathbb{R}^{p}\right)$ iff $h$ is integrable. The norm of $L^{1}\left([a, b] ; \mathbb{R}^{p}\right)$ is denoted by $\|\cdot\|_{1}$ and the norm of $L^{\infty}\left([a, b] ; \mathbb{R}^{p}\right)$ is $\|\cdot\|_{\infty}$.

Let $C^{*}([a, b] ; \mathbb{R})$ be the dual space of the continuous functions defined from $[a, b]$ to $\mathbb{R}$, denoted by $C([a, b] ; \mathbb{R})$, with supremum norm. The norm of $C^{*}([a, b] ; \mathbb{R})$ is denoted by $\|\mu\|_{T V}$. The set of elements in $C^{*}([a, b] ; \mathbb{R})$ which take nonnegative values on nonnegative valued functions in $C([a, b] ; \mathbb{R})$ is here denoted by $C^{\oplus}([a, b] ; \mathbb{R})$. For $\mu \in$ $C^{\oplus}([a, b] ; \mathbb{R}),\|\mu\|_{T V}=\int_{[a, b]} \mu(d t)$.

We use concepts from nonsmooth analysis. They are well known so we refrain from stating them here but we refer the reader to [9] for more information. Concerning nonsmooth analysis we use the following notation: $N_{S}^{L}\left(x^{*}\right)$ is the limiting normal cone to the set $S$ at $x^{*}$ (also known as Mordukhovich normal cone), $N_{S}^{C}\left(x^{*}\right)$ is the Clarke normal cone to $S$ at $x^{*}, \partial^{L} f\left(x^{*}\right)$ is limiting subdifferential or Mordukhovich subdifferential of $f$ at $x^{*}$ and $\partial^{C} f\left(x^{*}\right)$ is (Clarke) subdifferential of $f$ at $x^{*}$. If $f$ is Lipschitz continuous near $x^{*}$, the convex hull of the limiting subdifferential, co $\partial^{L} f\left(x^{*}\right)=\partial^{C} f\left(x^{*}\right)$.

\section{Minimum Time PROBlems With STATE CONSTRAINTS AND CONTROLS APPEARING LINEARLY}

In this section we deduce necessary conditions for a minimum time problem with vector valued state constraints using techniques provided in [9]. To maintain some of its generality we consider some data Lipschitz continuous.

Central to all this work is the following general minimum 
time optimal control problem with state constraints $(M)$ :

$$
\left\{\begin{array}{l}
\text { Minimize } t_{f} \\
\text { subject to } \\
\dot{\mathbf{y}}(t)=f(\mathbf{y}(t))+g(\mathbf{y}(t)) \mathbf{u}(t) \text { a.e. } t \in\left[0, t_{f}\right] \\
\mathbf{u}(t) \in \Omega \text { a.e. } t \in\left[0, t_{f}\right] \\
h(\mathbf{y}(t)) \leq 0 \text { for all } t \in\left[0, t_{f}\right] \\
\left(\mathbf{y}(0), \mathbf{y}\left(t_{f}\right)\right) \in\left\{\mathbf{y}_{0}\right\} \times E .
\end{array}\right.
$$

Here $t_{f}$ is a choice variable to be determined, $f: \mathbb{R}^{n} \rightarrow \mathbb{R}^{n}$, $h: \mathbb{R}^{n} \rightarrow \mathbb{R}^{K}$ are vector functions, $g: \mathbb{R}^{n} \rightarrow \mathbb{R}^{m \times n}$ is a matrix function, $\Omega \subset \mathbb{R}^{m}$ and $E \subset \mathbb{R}^{n}$ are closed sets and $\mathbf{y}_{0} \in \mathbb{R}^{n}$. We denote the $k$ components of $h$ by $h_{i}$ (with $i=1, \ldots, k)$.

A feasible process for $(M)$ is a triple $\left(t_{f}, \mathbf{y}, \mathbf{u}\right)$, satisfying the constraints of the problem with $t_{f}>0$, where $\mathbf{u}$ is a measurable control functions and $\mathbf{y}$, the state variable, is an absolutely continuous function. For any $t_{f}>0$, we identify a function $\mathbf{y}:\left[0, t_{f}\right] \rightarrow \mathbb{R}^{n}$ with its extension $\mathbf{y}_{e}$ to all $[0,+\infty$ [ by constant extrapolation of end values to the right: for example, if $\tilde{\mathbf{y}} \in \mathbb{R}^{n}$ and $t>t_{f}$, then $\left|\tilde{\mathbf{y}}-\mathbf{y}_{e}(t)\right|:=$ $\left|\tilde{\mathbf{y}}-\mathbf{y}\left(t_{f}\right)\right|$. In this way, given $t_{f}^{1}, t_{f}^{2}>0$ and two absolutely continuous functions $\mathbf{y}:\left[a, t_{f}^{1}\right] \rightarrow \mathbb{R}^{n}$ and $\mathbf{y}^{\prime}:\left[0, t_{f}^{2}\right] \rightarrow \mathbb{R}^{n}$ we define $\left\|\mathbf{y}-\mathbf{y}^{\prime}\right\|_{L^{\infty}}:=\left\|\mathbf{y}_{e}-\mathbf{y}_{e}^{\prime}\right\|_{L^{\infty}}$, where $\mathbf{y}_{e}$ and $\mathbf{y}_{e}^{\prime}$ are the extensions of $\mathbf{y}$ and $\mathbf{y}^{\prime}$. We say that $\left(\bar{t}_{f}, \overline{\mathbf{y}}, \overline{\mathbf{u}}\right)$ is a strong local minimizer for $(M)$ if there exists a $\varepsilon>0$ such that $\bar{t}_{f} \leq t_{f}$ over all feasible processes $\left(t_{f}, \mathbf{y}, \mathbf{u}\right)$ of $(M)$ satisfying $\left|t_{f}-\bar{t}_{f}\right|+\|\mathbf{y}-\overline{\mathbf{y}}\|_{L^{\infty}} \leq \varepsilon$.

As it is customary in the literature (see, for example, [9]), necessary conditions of optimality for $(M)$ can be derived reformulating the problem into another problem with fixed end time ${ }^{1}$, to which known necessary conditions are then applied. Assume the following hypotheses, which make reference to the process $\left(\bar{t}_{f}, \overline{\mathbf{y}}, \overline{\mathbf{u}}\right)$ and parameter $\varepsilon$, hold:

(H1) The set $E$ is closed and $\Omega \subset \mathbb{R}^{m}$ is a compact set.

(H2) There exist $\delta>0, K_{f}>0$ and $K_{g}>0$ such that,

$$
\left|\psi(\mathbf{y})-\psi\left(\mathbf{y}^{\prime}\right)\right| \leq K_{f}\left|\mathbf{y}-\mathbf{y}^{\prime}\right|
$$

for all $\mathbf{y}, \mathbf{y}^{\prime} \in \overline{\mathbf{y}}(t)+\delta B$ a.e. $t \in\left[0, \bar{t}_{f}\right]$, where $\psi=f$ and $\psi=g$.

(H3) The function $h$ is continuously differentiable ${ }^{2}$.

Assume that the data of our problem $(M)$ satisfy (H1) and (H2). Following the approach in [9], Chapter 8 (now with the aforementioned state constraints), we apply the nonsmooth Maximum Principle given by Theorem 9.3.1, multiple state constraint version, in [9] (page 331) for $(R)$ with reference to the strong local minimizer $(\bar{\tau}, \overline{\mathbf{y}}, \overline{\mathbf{u}}, \bar{\gamma})$. This leads to the following:

Theorem 2.1: Let $\left(\bar{t}_{f}, \overline{\mathbf{y}}, \overline{\mathbf{u}}\right)$ be a stong local minimizer for $(M)$. Assume that the assumptions (H1)-(H4) are satisfied. Then there exist an absolutely continuous function

\footnotetext{
${ }^{1}$ For non autonomous problems, the same can be done when the data is Lipschitz continuous with respect to time.

${ }^{2}$ We remark that the forthcoming analysis holds if Lipschitz continuity of $h$ is imposed instead of (H3). However, for our propose, (H3) is enough and it will somewhat simplify the exposition.
}

$p:\left[0, \bar{t}_{f}\right] \rightarrow \mathbb{R}^{n}$ and $\mu_{i} \in C^{\oplus}\left(\left[0, \bar{t}_{f}\right]\right), i=1, \ldots, k$ such that

$$
\operatorname{supp}\left\{\mu_{i}\right\} \subset\left\{s \in\left[0, \bar{t}_{f}\right]: h_{i}(\overline{\mathbf{y}}(s))=0\right\}
$$

and

$$
\begin{array}{cc}
\text { (a) } & \left(p, \mu_{1}, \mu_{2}, \ldots, \mu_{k}, \lambda\right) \neq(0,0, \ldots, 0,0), \\
(b) & -\dot{p}(t) \in \partial_{y}^{C} q(t) \cdot(f(\overline{\mathbf{y}}(t))+g((\overline{\mathbf{y}}(t)) \overline{\mathbf{u}}(t)), \\
(c) & q(t) \cdot(f(\bar{y}(t))+g(\overline{\mathbf{y}}(t)) \overline{\mathbf{u}}(t))= \\
& \max _{\mathbf{u} \in A} q(t) \cdot(f(\overline{\mathbf{y}}(t))+g(\overline{\mathbf{y}}(t)) \mathbf{u}), \\
(d) & -q(t) \cdot(f(\overline{\mathbf{y}}(t))+g((\overline{\mathbf{y}}(t)) \overline{\mathbf{u}}(t))=\lambda, \\
(e) & -q(T) \in N_{E}^{L}\left(\overline{\mathbf{y}}\left(\bar{t}_{f}\right)\right),
\end{array}
$$

where (b)-(d) hold for almost every a.e. $t \in\left[0, \bar{t}_{f}\right]$ and $q$ is

$$
q(t):=\left\{\begin{array}{cc}
p(t)+\int_{[0, t)} \sum_{i=1}^{k} \nabla h_{i}(\overline{\mathbf{y}}(t)) \mu_{i}(d s) \quad t<\bar{t}_{f} . \\
p\left(\bar{t}_{f}\right)+\int_{\left[0, \bar{t}_{f}\right]} \sum_{i=1}^{k} \nabla h_{i}(\overline{\mathbf{y}}(t)) \mu_{i}(d s) \quad t=\bar{t}_{f} .
\end{array}\right.
$$

III. AUV PROBLEM

We consider the problem of determining the path of an Autonomous Underwater Vehicle (AUV) on a horizontal plane to go from one point to a target set $T$ in the minimum time when currents are taken into account. We describe the motion using a simplified point mass model. For information on more realistic models of underwater vehicles we refer the reader to [2], for example.

A word of caution is need here. In what follows, and to keep our notation in agreement with the literature on AUVs, we use the variable $u$ to define the urge velocity of the vehicle while the control variable is denoted by $\mathbf{u}$.

We consider the following simplified kinematic model (see, for example, [8] and references therein):

$$
\begin{aligned}
\dot{x}(t) & =u(t) \cos (\phi(t))+v_{x}, \\
\dot{y}(t) & =u(t) \sin (\phi(t))+v_{y}, \\
\dot{\phi}(t) & =r(t),
\end{aligned}
$$

where $(x, y)$ denotes the position of the vehicle on the horizontal plane of constant depth, while $\phi$ represents its orientation, $u$ is the velocity of the vehicle, $r$ the angular velocity and $v=\left(v_{x}, v_{y}\right)$ the current velocity, which might depend on the position on the horizontal plane. The above equations are as in the well known Zermelo's problem. However, we couple these equations with a simplified dynamics equation of the form: $\dot{u}(t)=f(t)-K u(t)|u(t)|$, where $u$, the surge velocity of the vehicle, is a state and the thruster's force $f$ is an additional control. The term $-K u(t)|u(t)|$ depicts the quadratic drag force (see [4]) and, throughout this paper, we consider $K=1$. We assume that the velocity is limited $u(t) \in[0,2]$. Clearly, the velocity is not negative and so the term $-K u(t)|u(t)|$ in $\dot{u}(t)=f(t)-K u(t)|u(t)|$, can written simply by $K u^{2}(t)$. As in Zermelo's problem we consider that the velocity of ocean currents is known. For simplicity of the analysis, we assume that the velocity of the currents has components merely on the $x$ but depending on 
the $y$ position: $v(t)=(0.8 \tanh (y(t)), 0)$. To reflect the fact that the power of the thruster is limited and to bound the heading rate (making the model more realistic), we impose the control variables $(f, r)$ to take values in a given control set: $(f, r) \in[-5,5] \times[-\pi, \pi]$.

Our aim is to determine the minimum time $t_{f}$ needed to drive the vehicle from the point $\left(x_{0}, y_{0}\right)=(40,-2)$ to the target set

$$
T=\left\{(x, y): x^{2}+y^{2} \leq 0.05\right\} .
$$

The initial and final configurations of the vehicle are $\left(x_{0}, y_{0}, \phi_{0}, u_{0}\right)=(40,-2, \pi, 0)$ and $\left(x_{f}, y_{f}, \phi_{f}, u_{f}\right)=$ $\left(x_{1}, y_{1}, \pi, 0\right)$, where $\left(x_{1}, y_{1}\right) \in T$. Putting all together and considering $h(x, y, \phi, u)=\left(h_{1}(x, y, \phi, u), h_{2}(x, y, \phi, u)\right)$, with $h_{1}(x, y, \phi, u)=u-2$ and $h_{2}(x, y, \phi, u)=-u$, we now have the optimal control problem:

$$
\begin{aligned}
& \text { Minimize } \quad t_{f} \\
& \text { subject to } \\
& \quad \dot{x}(t)=u(t) \cos (\phi(t))+0.8 \tanh (y(t)) \text { a.e., } \\
& \dot{y}(t)=u(t) \sin (\phi(t)) \text { a.e., } \\
& \dot{\phi}(t)=r(t) \text { a.e., } \\
& \quad \dot{u}(t)=f(t)-u(t) .|u(t)| \text { a.e., } \\
& (x(0), y(0), \phi(0), u(0))=[40,-2, \pi, 0], \\
& \left(x\left(t_{f}\right), y\left(t_{f}\right), \phi\left(t_{f}\right), u\left(t_{f}\right)\right) \in T \times\{(\pi, 0)\} \\
& \quad h(x(t), y(t), \phi(t), u(t)) \leq 0 \text { for all } t \in\left[0, t_{f}\right] \\
& (f, r) \in[-5,5] \times[-\pi, \pi] .
\end{aligned}
$$

Problem $(P)$ is a minimum time problem with control appearing linearly in the dynamics and with state constraints. Here $\mathbf{y}=(x, y, \phi, u)$ is the state variable, $\mathbf{u}=(f, r)$ is the control variable and $\mathbf{p}=\left(p_{x}, p_{y}, p_{\phi}, p_{u}\right)$ is the adjoint multiplier. It is then a simple matter to see that $(P)$ is in the form of $(M)$ for the appropriate choice of $f$ and $g$. The Hamiltonian function for $(P)$ is then

$$
\begin{gathered}
H(\mathbf{y}, \mathbf{p}, \mathbf{u})= \\
p_{x} u \cos (\phi)+0.8 p_{x} \tanh (y)+p_{y} u \sin \phi+p_{\phi} r+p_{u}\left(f-u^{2}\right) .
\end{gathered}
$$

Assume that $\left(\bar{t}_{f}, \overline{\mathbf{y}}=(\bar{x}, \bar{y}, \bar{\phi}, \bar{u}), \overline{\mathbf{u}}=(\bar{f}, \bar{r})\right)$ is a strong local solution of $(P)$. Since the data of $(P)$ satisfies the conditions under which Theorem 2.1 holds, we deduce that there exist absolutely continuous function $\mathbf{p}$, Borel regular measures $\mu_{1}, \mu_{2}$ and $\lambda \geq 0$, not all 0 , such that

$$
\begin{aligned}
& \dot{p}_{x}=0, \\
& \dot{p}_{y}=-0.8 p_{x} \frac{1}{\cosh ^{2}(\bar{y})}, \\
& \dot{p}_{\phi}=p_{x} \bar{u}(t) \sin (\bar{\phi})-p_{y} \bar{u} \cos (\bar{\phi}), \\
& \dot{p}_{u}=-p_{x} \cos (\bar{\phi})-p_{y} \sin (\bar{\phi})+2 q_{u}(t) \bar{u}, \\
& p_{\phi} \bar{r}+q_{u} \bar{f}=\max _{(f, r) \in[-5,5] \times[-\pi, \pi]} p_{\phi} r+q_{u} f,
\end{aligned}
$$

(with all the above holding for almost every $t \in\left[0, \bar{t}_{f}\right]$ ) together with

$$
\begin{gathered}
-\lambda=p_{x} \bar{u} \cos (\bar{\phi})+0.8 p_{x} \tanh (\bar{y})+p_{y} \bar{u} \sin (\bar{\phi}) \\
+p_{\phi} \bar{r}+q_{u}\left(\bar{f}-\bar{u}^{2}\right)
\end{gathered}
$$

$$
\left(-p_{x}\left(\bar{t}_{f}\right),-p_{y}\left(\bar{t}_{f}\right)\right) \in N_{T}^{L}\left(\bar{x}\left(\bar{t}_{f}\right), \bar{y}\left(\bar{t}_{f}\right)\right),
$$

(in (3)-(8) we drop the $t$ in all variable to simplify the notation) where

$q_{u}(t)=\left\{\begin{array}{cc}p_{u}(t)+\int_{[0, t)} \mu_{1}(d s)-\int_{[0, t)} \mu_{2}(d s), & t<\bar{t}_{f} . \\ p_{u}\left(\bar{t}_{f}\right)+\int_{\left[0, \bar{t}_{f}\right]} \mu_{1}(d s)-\int_{\left[0, \bar{t}_{f}\right]}^{\mu_{2}(d s),} & t=\bar{t}_{f},\end{array}\right.$

and $\operatorname{supp}\left\{\mu_{i}\right\}$ are subsets of the active sets of $h_{i}, i=1,2$.

Next we extract information about the optimal solution from the above conditions. Before proceeding it is worth to recall the following facts:

F1) Since our final state $\mathbf{y}\left(\bar{t}_{f}\right)$ is constrained, we cannot, a priori, assume that $\lambda=1$.

F2) The left-continuous function of bounded variation $\nu(t)=\int_{[0, t)} \mu_{1}(d \sigma)$ can be further decomposed uniquely as

$$
\nu(t)=\nu^{a}(t)+\nu^{s}(t)+\nu^{j}(t)
$$

where $\nu^{a}(t)=\int_{0}^{t} \nu^{a}(s) d s$ is an absolutely continuous function, $\nu^{s}(t)=\int_{[0, t)} \mu_{2}^{s}(d s)$ is a continuous but not absolutely continuous function and $\nu^{j}$ is a pure jump function with at most a countable number of jumps. Let $\tau_{i}$ denote the points of discontinuity of $\nu$.

F3) A state constraint $h(\overline{\mathbf{y}}(t)) \leq 0$ has a boundary interval $\left[t_{0}^{b}, t_{1}^{b}\right]$ if $h(\overline{\mathbf{y}}(t))=0$ for all $t \in\left[t_{0}^{b}, t_{1}^{b}\right]$ and the points $t_{0}^{b}$ and $t_{1}^{b}$ are called junctions points if $h(\overline{\mathbf{y}}(t))<0$ for $t$ in neighbourhoods of those point, on the left of $t_{0}^{b}$ and on the right of $t_{1}^{b}$ (in this case $t_{0}^{b}$ is called an entry point and $t_{1}^{b}$ an exit point), it has a contact point if there exist $\sigma_{i}$ and $\delta>0$ such that $h\left(\overline{\mathbf{y}}\left(\sigma_{i}\right)\right)=0$ and $h(\overline{\mathbf{y}}(t))<0$ for all $t \in\left(\left[\sigma_{i}-\delta, \sigma_{i}[\cup] \sigma_{i}, \sigma_{i}+\delta[) \cap\left[0, \bar{t}_{f}\right]\right.\right.$ and, finally, it has an interior interval $\left[t_{0}^{i}, t_{1}^{i}\right]$ if $h(\overline{\mathbf{y}}(t))<0$ for all $\left.t \in\right] t_{0}^{i}, t_{1}^{i}[$.

We are now in position to turn to our problem. Problem $(P)$ has two state inequality constraints that are never simultaneously active. Taking into account the physical meaning of the problem it is to be expected that $h_{2}$ will be active only at the extreme points $t=0$ and $t=\bar{t}_{f}$ ( since $\bar{u}(0)=\bar{u}\left(\bar{t}_{f}\right)$ ) and, consequently, it is reasonable to expect that there is no boundary interval for $h_{2}$. The same, however, cannot be said about $h_{1}$.

A word of caution in this regard is called for. Indeed, the fact that $h_{2}$ is active at $t=0$, because $\bar{u}(0)=0$, could undermine the applicability of the necessary conditions (a)-(d) of Theorem 2.1 since it is well known that when the initial state is on the boundary of a state constraint, the maximum principle may fail to provide any information about the solution, i.e., the maximum principle may be degenerate. In such situations, nondegenerate forms of the maximum principle have been established in the literature under different constraints qualifications (see, for example, [5] and references within). However, it is a simple matter to see that the inward pointing velocity condition (see in [5]) holds: there exists a control $\mathbf{u}(t)=(\pi, f)$, where $f \in[1,5[$ (for example), such that, for $t$ in a neighbourhood of 0 we have $\nabla h_{2}(\overline{\mathbf{y}}(0)) \cdot(f(\overline{\mathbf{y}}(0))+g(\overline{\mathbf{y}}(0)) \mathbf{u}(t))=-f<5$. 
Since the control appears linearly in the Hamiltonian, we know that the optimal solution of our problem is a concatenation of bang and singular arcs. We say that $\left[t_{0}^{r_{s}}, t_{1}^{r_{s}}\right]$ is a singular interval for the control component $r$ if $\bar{r}(t) \in$ ]$-\pi, \pi[$ for $t \in] t_{0}^{r_{s}}, t_{1}^{r_{s}}$ [ and that $\left[t_{0}^{f_{s}}, t_{1}^{f_{s}}\right]$ is a singular interval for the control component $f$ if $\bar{f}(t) \in]-5,5[$ for $t \in] t_{0}^{f_{s}}, t_{1}^{f_{s}}[$. Let us define the switching function as

$$
\psi(\overline{\mathbf{y}}(t), \mathbf{p}(t))=\left[\begin{array}{c}
\psi_{f}(\overline{\mathbf{y}}(t), \mathbf{p}(t)) \\
\psi_{r}(\overline{\mathbf{y}}(t), \mathbf{p}(t))
\end{array}\right]=\left[\begin{array}{c}
q_{u}(t) \\
p_{\phi}(t)
\end{array}\right] .
$$

Evaluating $\psi$ along the optimal trajectory $\overline{\mathbf{y}}$ and for a certain $\mathbf{p}, \psi$ depends on $t$. Thus we write merely $\psi(t)$ when the dependence on $\overline{\mathbf{y}}$ and $\mathbf{p}$ is clearly understood. From (7), we get the following control laws: $\bar{f}(t)=-5$ if $\psi_{f}(t)>0$; $\bar{f}(t)=f^{s}(t)$ if $\psi_{f}(t)=0 ; \bar{f}(t)=5$ if $\psi_{f}(t)<0 ; \bar{r}(t)=$ $-\pi$ if $\psi_{r}(t)>0 ; \bar{r}(t)=r^{s}(t)$ if $\psi_{r}(t)=0 ; \bar{r}(t)=\pi$ if $\psi_{r}(t)<0$. Both $f^{s}$ and $r^{s}$, the values of the singular controls, represent values in $]-5,5[$ and $]-\pi, \pi[$.

Preparing for the validation of numerical results, we consider a situation that suggests itself from the physiscal meaning of our problem. Let us then suppose that there exists no boundary interval for $h_{2}$ and there exist only one boundary interval for $h_{1},\left[t_{0}^{b}, t_{1}^{b}\right] \subset\left[0, \bar{t}_{f}\right]$, where $\left.t_{0}^{b} \in\right] 0, \bar{t}_{f}[$ is the first point where $h_{1}(\overline{\mathbf{y}}(t))=0$ and $t_{1}^{b}$ is the last point where $h_{1}(\overline{\mathbf{y}}(t))=0$. We also assume that there is not other contact points for both $h_{1}$ and $h_{2}$ besides those mentioned above. In such situation we deduce that:

(1) for all $t \in\left[t_{0}^{b}, t_{1}^{b}\right]$ we have $\bar{u}(t)=2$. Consequently $\dot{\bar{u}}(t)=$ 0 and $\bar{f}(t)=4$ for $t \in] t_{0}^{b}, t_{1}^{b}[$. Thus $] t_{0}^{b}, t_{1}^{b}[\subseteq] t_{0}^{f_{s}}, t_{1}^{f_{s}}[$ and since $\bar{f}$ is singular, we have $q_{u}(t)=0$ for all $\left.t \in\right] t_{0}^{b}, t_{1}^{b}[$. Recall that in this situation we have ${ }^{3}$

$$
q_{u}(t)=p_{u}(t) \text { for } t \in\left[0, t_{0}^{b}[,\right.
$$

and

$$
\left.0=p_{u}(t)+\int_{[0, t)} \mu_{1}(d \sigma) \text { for } t \in\right] t_{0}^{b}, t_{1}^{b}[.
$$

(2) Using the notation introduced in F2, we have $\nu^{j}\left(\tau_{i}\right)=$ $\mu_{1}\left(\left\{\tau_{i}\right\}\right)=\nu\left(\tau_{i}^{+}\right)-\nu\left(\tau_{i}^{-}\right)$and $\nu^{j}(t)=0$ if $t \neq \tau_{i}$. Since $\nu(t)=0$ for $t<t_{0}^{b}$ and $q_{u}(t)=0$ for $\left.t \in\right] t_{0}^{b}, t_{1}^{b}[$ we have two possibilities: (i) either $\tau_{1}=t_{0}^{b}$ and then $\mu_{1}\left(\left\{\tau_{1}\right\}\right) \neq 0$ and $0=p_{u}(t)+\mu_{1}\left(\left\{\tau_{1}\right\}\right)+\nu^{a}(t)$, (ii) or, $t_{0}^{b}$ is not a point of discontinuity of $\nu$ and then we have $\mu_{1}\left(\left\{\tau_{1}\right\}\right)=0$ and $p_{u}(t)=\nu^{a}(t)$.

(3) Doing a similar analysis at $t_{1}^{b}$ we conclude from the above that, then at $t_{0}^{b}$ and at $t_{1}^{b}$ we have

$q_{u}\left(t_{0}^{b+}\right)-q_{u}\left(t_{0}^{b-}\right)=\mu_{1}\left\{t_{0}^{b}\right\}, q_{u}\left(t_{1}^{b+}\right)-q_{u}\left(t_{1}^{b-}\right)=\mu_{1}\left\{t_{1}^{b}\right\}$.

If $t_{0}^{b}$ (or $t_{1}^{b}$ ) is not a discontinuity point of $q_{u}$, then $\mu_{1}\left(\left\{t_{0}^{b}\right\}\right)=0\left(\mu_{1}\left(\left\{t_{1}^{b}\right\}\right)=0\right)$.

(4) Moreover, $q_{u}$ has no jumps inside the boundary interval and $\nu^{a}(t)=0$ if $t \in\left[0, t_{0}^{b}\right] \cup\left[t_{1}^{b}, \bar{t}_{f}\right]$.

(5) We can then write

$$
p_{u}(t)=\left\{\begin{array}{l}
q_{u}(t) \text { if } t \in\left[0, t_{0}^{b}[\right. \\
\left.\left.q_{u}\left(t_{0}^{b-}\right)-\nu^{a}(t) \text { if } t \in\right] t_{0}^{b}, t_{1}^{b}\right] \\
\left.\left.q_{u}(t)+q_{u}\left(t_{0}^{b-}\right)+q_{u}\left(t_{1}^{b+}\right) \text { if } t \in\right] t_{1}^{b}, \bar{t}_{f}\right] .
\end{array}\right.
$$

\footnotetext{
${ }^{3}$ The fact that the measure $\mu_{1}$ is assumed 0 here is because of the nondegeneracy of our maximum principle.
}

(6) Based on the physical meaning of $r$, it is not unreasonable to expect that $r$ takes singular values except at small neighbourhoods of 0 and $\bar{t}_{f}$ and when $\bar{u}(t) \neq 0$. We then foreseen the existence of a singular interval $\left[t_{0}^{r}, t_{1}^{r}\right]$, with $0<t_{0}^{r}<t_{1}^{r}<\bar{t}_{f}$ and that $\left[t_{0}^{b}, t_{1}^{b}\right] \subset\left[t_{0}^{r}, t_{1}^{r}\right]$. Seeking a closed form for $r^{\text {sing }}$ we set $\psi_{r}(t)=0$ for $t \in\left[t_{0}^{b}, t_{1}^{b}\right]$. We have $\frac{d}{d t} \psi_{r}(t)=0$ for $\left.t \in\right] t_{0}^{r}, t_{1}^{r}[$ and, using this equality, the fact that $\bar{u}(t) \neq 0$ and imposing that $\frac{d^{2}}{d t^{2}} \psi_{r}=0$ we get

$$
r\left(p_{x}(t) \cos (\bar{\phi}(t))+p_{y}(t) \sin (\bar{\phi}(t))\right)-\dot{p}_{y}(t) \cos (\bar{\phi}(t))=0 .
$$

If

$$
\left.p_{x}(t) \cos (\bar{\phi}(t))+p_{y}(t) \sin (\bar{\phi}(t)) \neq 0 \text { for } t \in\right] t_{0}^{r}, t_{1}^{r}[,
$$

then the generalized Legendre-Clebsch condition $\frac{\partial}{\partial r}\left(\frac{d^{2}}{d t^{2}} \psi_{r}\right) \neq 0$ holds allowing us to deduce that

$$
r^{s}(\overline{\mathbf{y}}(t))=-0.8 \cos ^{2}(\bar{\phi}(t)) \frac{1}{\cosh ^{2}(\bar{y}(t))} .
$$

The expression (14) holds provided that $\cos \bar{\phi}(t) \neq 0$ for $t \in] t_{0}^{r}, t_{1}^{r}[$.

(7) It remains to know if there exists a singular interval for $f$ outside or containing the boundary interval and what value would $f$ take there. However, an analysis for $\psi_{f}$ analogous to the one in (6) above is not possible since we have $\frac{\partial}{\partial f}\left(\frac{d^{2}}{d t^{2}} \psi_{f}\right)=q_{u}(t)=0$ and so it does not provide a test for optimality of this arc.

\section{Numerical Results}

We now present the numerical solution of problem $(P)$ as defined in section 3 with $T$ as in (2). We use the Euler Method to solve AUV's problem with 10000 grid nodes. As mentioned in the Introduction, we use the Applied Modeling Programming Language, AMPL, as the interface with version 3.8.0 of IPOPT. When the trapezoidal method is used instead of the Euler method the computed controls and state exhibit small but nevertheless significant numerical chattering at the switching points.

We obtain $\bar{t}_{f}=14.944$ as the minimum time. We provide the computed values of the endpoints of the state $x$ and $y$ and the initial values of the multipliers in the table below.

Numerical results for $(x(0), y(0))=(40,-2)$ :

$$
\begin{array}{ll}
\bar{t}_{f}=14.944, & \\
x\left(\bar{t}_{f}\right)=0.15625, & y\left(\bar{t}_{f}\right)=-0.15995 \\
p_{x}(0)=-0.35811, & p_{y}(0)=-0.05657, \\
p_{\phi}(0)=0.000112, & p_{u}(0)=0.14208 .
\end{array}
$$

In figure 1 we present the trajectory in the horizontal plane and, in figure 2 , the computed optimal controls .

The controls $r$ and $f$ are both discontinuous and bangsingular-bang with two switching points. The control $\bar{r}$ has switching points $t_{1} \approx 0.05$ and $t_{2} \approx 14.7$, the switching points of $\bar{f}$ are approximately 0.64 and 14.62 The computed state variables are plotted in figure 3 . The computed values show that the singular interval of $\bar{f}$ coincides with the boundary interval of the state constraint $u-2 \leq 0$ as shown 


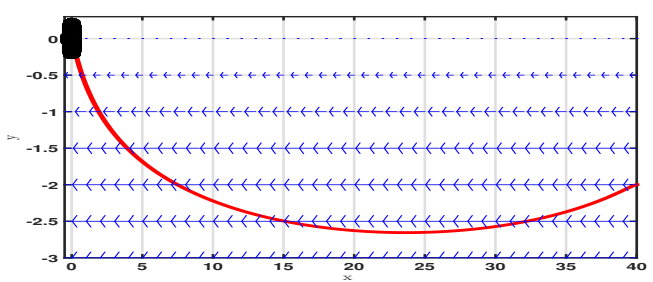

Fig. 1. Minimum time trajectory for the AUV for $(x(0), y(0))=$ $(40,-2)$.

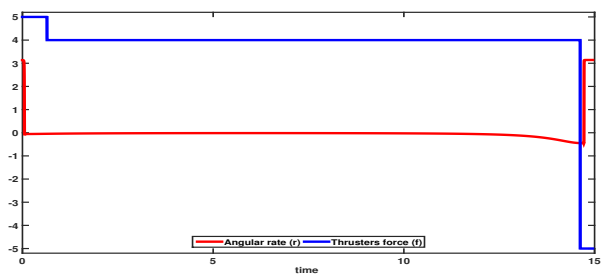

Fig. 2. Optimal controls $r$ and $f$ the AUV for $(x(0), y(0))=$ $(40,-2)$.
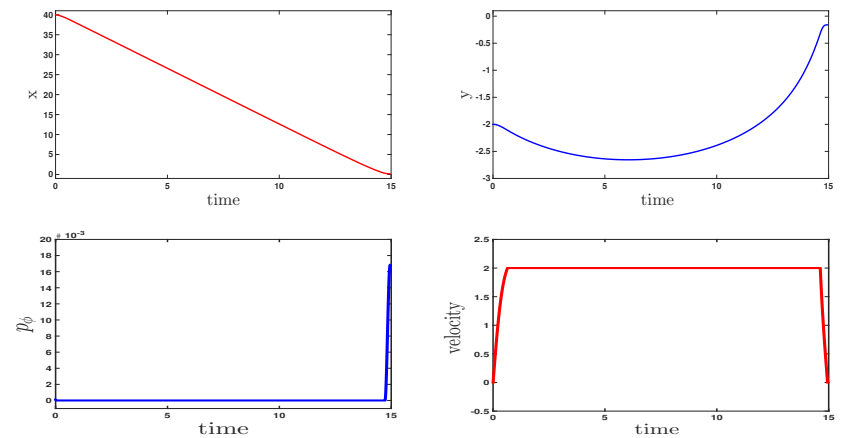

Fig. 3. State variables for the AUV problem. Scales on the vertical axes are different.

in figure 4, where the graph of the state variable $u$ is plotted together with the graph of the control $\bar{f}$. Our analysis shows

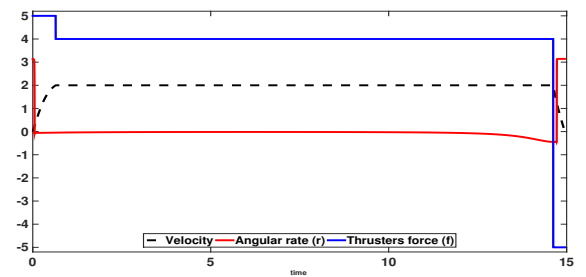

Fig. 4. The computed values of $u$ plotted together with those of $f$.

that the multiplier $q_{u}$ must be 0 along the boundary arc. This is confirmed by the numerical values as shown in figure 5 where we present the computed multipliers $p_{x}, p_{y}, p_{\phi}$ and $q_{u}$. Since the computed solution reaches the boundary of $T$ in the fourth quadrant, we deduce from (9) and (3) that we should have $p_{x}(t)=p_{x}\left(\bar{t}_{f}\right)<0$ and $p_{y}\left(\bar{t}_{f}\right)>0$ : this is confirmed by the computed values as seen in figure 5 .
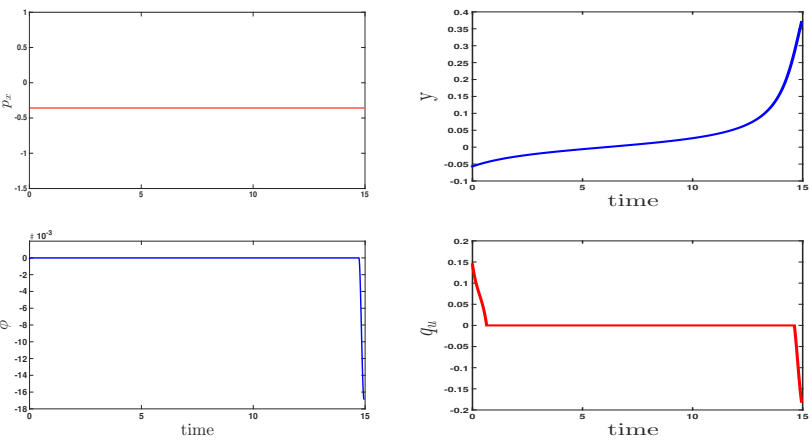

Fig. 5. State variables for the AUV problem. Scales on the vertical axes are different.

A careful analysis of the computed values of $\phi, p_{\phi}$ and $p_{x}$ confirms that (13) holds. Confronting the numerical expression of the analytical value of the $r^{\mathrm{s}}$ in (14) with the computed value of $\bar{r}$, shown in 6 , we see they coincide. The numerics also show that the multiplier $\lambda$ is 1 as shown in the right side of figure 6 where the Hamiltonian is plotted verifying (apart from some residual numerical chattering) (8).

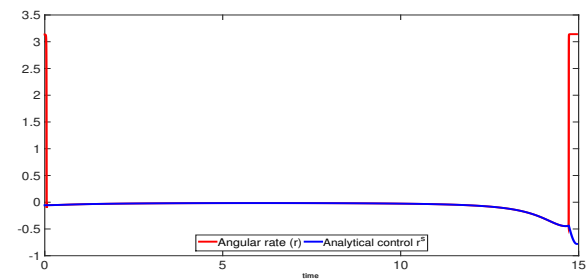

Fig. 6. The graph of the analytic value of $r$ in (14) and the numerical value of the optimal control $r$ : they match.

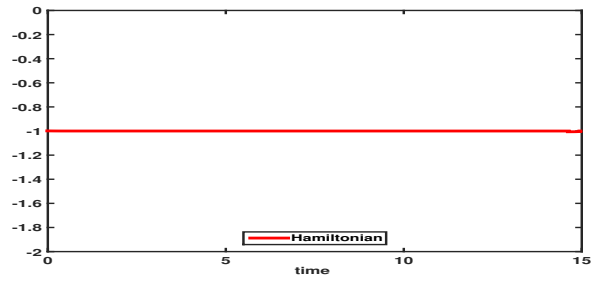

Fig. 7. The computed value of the Hamiltonian that is equal to $-\lambda$ (see (8)): the computed $\lambda$ is indeed 1 .

With the computed values we can determine the values of the bounded variations functions (see, in this respect, F2 in section III)

$\mu_{1}\left(\left\{t_{0}^{b}\right\}\right) \approx 0.0011, \mu_{1}\left(\left\{t_{1}^{b}\right\}\right) \approx 0.0014, \mu_{2}(\{T\}) \approx 0.0011$

and to $p_{u}$. Those atoms are responsible for the discontinuity of $q_{u}$ at the boundary points and the final time. To finalize this section, we emphasize the fact that we use the constraint $u(t) \in[0,2]$ to compute all our numeric findings. However, running the codes with $u(t) \in[-2,2]$ produces exactly the same values apart form the value $\mu_{2}$ (that it is not present) and, consequently, $q_{u}$ has no discontinuity in the last instant. 


\section{Sensitivity With Respect to Initial Conditions}

To test the sensivity of the solution we calculate the trajectories for different for different values of $(x(0), y(0))$, keeping $(\phi(0), u(0))=(\pi, 0)$. Graphs of various trajectories are shown in figure 8 . The profile of the trajectories do

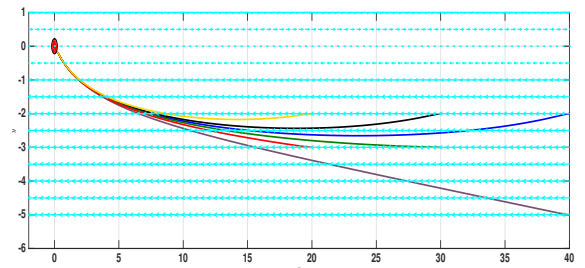

Fig. 8. Minimum time trajectory for the AUV for various values of $(x(0), y(0))$.

not change. Observe that in the cases studied, the vehicle is initially oriented towards the left (the $y$ axe). It is then to be expected that the vehicle moves forward and never backwards to get to the target. The same may not hold if we change the initial orientation of the vehicle as we illustrate next. To do so we consider the following initial and final conditions:

(O1) $(x(0), y(0), \phi(0), u(0))=(0,0,0,0)$,

(O2) $\left(x\left(t_{f}\right), y\left(t_{f}\right), \phi\left(t_{f}\right), u\left(t_{f}\right) \in T_{1} \times\{(0,0)\}\right.$ where $T_{1}=$ $\left\{(x, y):(x-40)^{2}+(y+2)^{2} \leq 0.05^{2}\right\}$

(O3) $u(t) \in[-2,2]$.

The vehicle now starts at the origin oriented towards the west and should arrive at a small ball around the pint $(40,-2)$, placed in the east. The computed minimum time is now 16.341 .
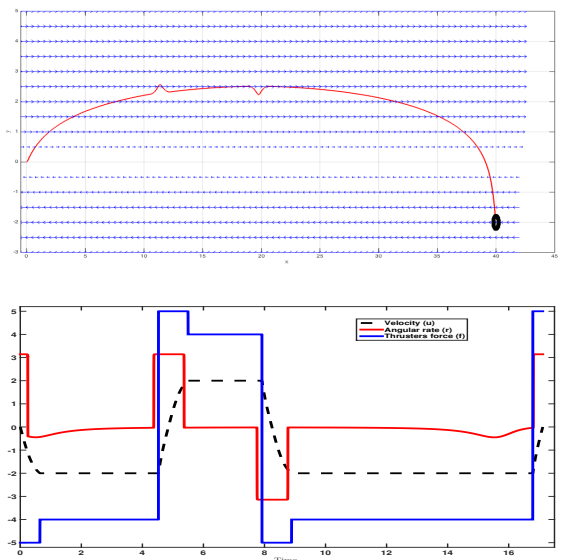

Fig. 9. For the minimum time problem when (O1)-(O3) are enforced, trajectory of the AUV in the $x 0 y$ plane in the top and the graphs of velocity $u$ and the controls $r$ e $f$ in the bottom.

Clearly from figure 9 the AUV moves backward for a while, then it turns forwards to continue with forward movement for a brief period, returning to the backward movement so that it can stop at the target with the prescribed orientation. The velocity $u$ has three boundary arcs: we have $u(t)=-2$ for (approximately) $t \in[0.64,4.53]$ and for $t \in[8,89,16,79]$ while $u(t)=2$ for $t \in[5.50,7.91]$.

Finally, we observe that abrupt changes in the controls as those computed in the present paper, are generally undesirable because they may cause equipment malfunctions. To avoid such situation we propose to add a running cost to the objective of $(P)$. Instead of minimizing $t_{f}$ we propose to minimize

$$
t_{f}+\int_{0}^{t_{f}}\left(f^{2}(t)+r^{2}(t)\right) d t .
$$

For $(P)$ equipped with this new cost, the computed values show that the minimum cost may be highly increased. With the initial and final conditions described in previous section we get for minimum time 31.0311 that is slightly greater than twice the minimum time obtained before.

\section{ACKNOWLEDGMENT}

The first author would like to thank the support of SFRH/BSAB/114265/2016 funds through FCT, Project POCI-01-0145-FEDER-006933 - SYSTEC and PTDC/EEIAUT/2933/2014, TOCCATTA. These two last ones are funded by FEDER funds through COMPETE2020 - POCI and by national funds through FCT - Fundacao para a Ciencia e a Tecnologia. She also thanks ENSTA- Paris Tech and her host there, Hasnaa Zidani, for the very warm welcome and stay. The second authors thanks the Faculdade de Engenharia da Universidade do Porto where she spent six months in 2015 -2016 for the warm way she was received there. The third author thanks the financial support of INESC-TEC.

\section{REFERENCES}

[1] M. Chyba, H. Maurer, H. Sussmann, H. and G Vossen, Underwater vehicles: The minimum time problem. In Proceedings of the 43th IEEE Conf. on Decision and Control (2004).

[2] M. Chyba and T. Haberkorn and R. N. Smith Song K. and Choi, Autonomous Underwater Vehicles: Development and Implementation of Time and Energy Efficient Trajectories1, Ship Technology Research, Maney Publishing Suite 1C, Leeds, UK (2015).

[3] P. Falugi, E. Kerrigan and E. van Wyk, Imperial College London Optimal Control Software User Guide (ICLOCS), Department of Electrical and Electronic Engineering, Imperial College London, London, England, UK (2010).

[4] Thor Fossen, Guidance and Control of Ocean Vehicles, John Wiley \& Sons Ltd, 1994

[5] F. Fontes and H. Frankowska, Normality and Nondegeneracy for Optimal Control Problems with State Constraints, Journal of Optimization Theory and Applications, 166, 1, pp. 115-136 (2015).

[6] Nikolai P. Osmolovskii and Helmut Maurer, Applications to Regular and Bang-Bang Control: Second-Order Necessary and Sufficient Optimality Conditions in Calculus of Variations and Optimal Control, SIAM Advances in Design and Control, 24, 2013.

[7] F. Rampazzo and R. Vinter, A theorem on existence of neighbouring trajectories satisfying a state constraint, with applications to optimal control, IMA J. Math. Control \& Information, 16 (4), (1999) 335-351.

[8] J. E. Silva and João Sousa, Models for Simulation and Control of Underwater Vehicles, New Approaches in Automation and Robotics, pp. 197-207, Editor: Harald Aschemann, I-Tech Education and Publishing (2008).

[9] Richard Vinter, Optimal Control, Birkhäuser, Boston, 2000.

[10] Andreas Wächter and Lorenz T. Biegler, On the implementation of an interior-point filter line-search algorithm for large-scale nonlinear programming, Mathematical Programming, 106 (2006), 25-57. 